\title{
Structural basis for transcription reactivation by RapA
}

\author{
Bin Liu" ${ }^{a, 1}$, Yuhong Zuo ${ }^{a, 1}$, and Thomas A. Steitz ${ }^{a, b, c, 2}$ \\ aDepartment of Molecular Biophysics and Biochemistry, ${ }^{\mathrm{b}}$ Howard Hughes Medical Institute, and 'Department of Chemistry, Yale University, New Haven, \\ CT 06520
}

Edited by Wei Yang, National Institutes of Health, Bethesda, MD, and approved January 7, 2015 (received for review September 4, 2014)

RNA polymerase (RNAP) loses activity during transcription as it stalls at various inactive states due to erratic translocation. Reactivation of these stalled RNAPs is essential for efficient RNA synthesis. Here we report a 4.7-Å resolution crystal structure of the Escherichia coli RNAP core enzyme in complex with ATPase RapA that is involved in reactivating stalled RNAPs. The structure reveals that RapA binds at the RNA exit channel of the RNAP and makes the channel unable to accommodate the formation of an RNA hairpin. The orientation of RapA on the RNAP core complex suggests that RapA uses its ATPase activity to propel backward translocation of RNAP along the DNA template in an elongation complex. This structure provides insights into the reactivation of stalled RNA polymerases and helps support ATP-driven backward translocation as a general mechanism for transcriptional regulation.

RNA polymerase | RapA | DNA translocase | transcription |

backtranslocation

$\mathbf{R}^{\mathrm{s}}$ NA polymerase (RNAP) translocates along DNA while synthesizing RNA during transcription. For continuous RNA synthesis, RNA polymerase moves from the pretranslocation position to the posttranslocation position after incorporation of each nucleotide $(1,2)$. This forward translocation removes the transcript from the RNAP active site, which allows the binding of the next NTP. Although details about the translocation mechanism are still under debate, it is known that RNAP makes frequent pauses during the repetitive nucleotide additions (3-6). Some of the initial pauses rearrange to longer-lived pausing states involving hyperforward translocation (hypertranslocation) or backward translocation (backtranslocation) (backtracking) and lead to an arrest of RNA synthesis. Hypertranslocation, which is promoted by nascent RNA hairpin formation or ATPases, has been suggested as a general mechanism for transcription termination (7, 8 ), whereas backtracking, albeit a mechanism for transcriptional proofreading (9), is a major cause of transcriptional inactivation (10). Assisted backtracking has only been suggested recently for RNAPs that are blocked by DNA damage (11).

RapA is an abundant RNAP-associated protein of $110-\mathrm{kDa}$ molecular weight with ATPase activity (12). It forms a stable complex with the RNAP core enzyme, but not with the holoenzyme $(13,14)$. The ATPase activity of RapA increases upon its binding to RNAP (12). It has been shown that RapA reactivates stalled RNAPs and stimulates RNA synthesis in vitro $(13,15)$. We have determined the crystal structure of RapA in complex with the Escherichia coli RNAP core at $4.7-\AA$ resolution. The orientation of RapA relative to RNAP in our complex suggests that RapA reactivates RNAPs through an ATP-driven backtranslocation mechanism.

\section{Results and Discussion}

Overall Structure of RNAP Core in Complex with RapA. The complex of RapA bound to RNAP core was crystallized in the presence of a DNA-RNA hybrid (Fig. 1, Fig. S1, and Table 1). There are two copies of the complex per asymmetric unit with one RapA molecule associated with each RNAP core. The DNA-RNA hybrid contains a 9-bp perfect duplex and has its RNA $3^{\prime}$ end positioned in the posttranslocation position in the active site channel. It appears that this hybrid is essential for the crystallization of this complex, possibly because it stabilizes the RNAP core in a closed conformation similar to that of an elongation complex (16).

RapA is a putative double-stranded DNA (dsDNA) translocase of the Swi2/Snf2 protein family, whose members have been found to mobilize various nucleic acid-protein complexes $(17,18)$. A previous study showed that RapA consists of an $\mathrm{N}$-terminal domain (NTD), an ATPase core, a spacer domain, and a C-terminal domain (CTD) (Fig. $2 A$ and Fig. S2) (13). The ATPase core of RapA contains four structural domains: RecA-like 1A and 2A domains that are conserved for many ATP-dependent nucleic acid-processing enzymes, and enzyme-specific Swi2/Snf2 domains $1 \mathrm{~B}$ and $2 \mathrm{~B}$. $1 \mathrm{~A}$ and $1 \mathrm{~B}$ domains of RapA form one lobe of the ATPase core, whereas $2 \mathrm{~A}$ and $2 \mathrm{~B}$ domains form the other. The ATPase active site lies at the interface between the two lobes. The CTD and the spacer domain of RapA are tightly associated with the $1 \mathrm{~A} / 1 \mathrm{~B}$ lobe, whereas the NTD, which consists of two antiparallel $\beta$-strand folds, connects to the $1 \mathrm{~A} / 1 \mathrm{~B}$ lobe via a flexible linker.

Whereas RapA adopts essentially the same conformation in this complex as that observed previously in the free form, the association of RapA with RNAP causes a large displacement of its $\beta$-flap tip, which is a short alpha helix that lies at the exit of the RNA channel (Fig. 2B). This flap tip helix (FTH) is connected to the rest of the flap domain via flexible linkers and is the binding site of the CTD of the $\sigma$-factor in an RNAP holoenzyme (19-21). The binding site of RapA overlaps with that of the $\sigma$-CTD, which explains why RapA binds only to the RNAP core enzyme, but not to the holoenzyme.

RapA Modifies the RNA Exit Channel. In this complex, RapA binds at the exit of the RNA channel of the RNAP core and makes

\section{Significance}

Transcription is the first and most regulated step of gene expression. During transcription, RNA polymerase (RNAP) translocates along DNA while processively synthesizing RNA molecules hundreds of nucleotides long. Excessive translocation in either direction halts RNA synthesis. Here, we present a structure of the Escherichia coli RNA polymerase in complex with ATPase RapA, a large DNA translocase that is involved in transcriptional reactivation. The structural insights gained from this study suggest an alternative mechanism for transcription regulation in which backward translocation (backtranslocation) might be promoted by a DNA translocase and also lead us to propose a model for how RapA reactivates RNA polymerases and stimulates transcription. This active backtranslocation proposed here could be a general mechanism for regulating transcription and transcription-related processes.

Author contributions: B.L., Y.Z., and T.A.S. designed research; B.L. and Y.Z. performed research; B.L. and Y.Z. analyzed data; and B.L., Y.Z., and T.A.S. wrote the paper.

The authors declare no conflict of interest.

This article is a PNAS Direct Submission.

Data deposition: The atomic coordinates and structure factors have been deposited in the Protein Data Bank, www.pdb.org (PDB ID code 4S20).

${ }^{1}$ B.L. and Y.Z. contributed equally to this work.

${ }^{2}$ To whom correspondence should be addressed. Email: thomas.steitz@yale.edu.

This article contains supporting information online at www.pnas.org/lookup/suppl/doi:10 1073/pnas.1417152112/-/DCSupplemental. 


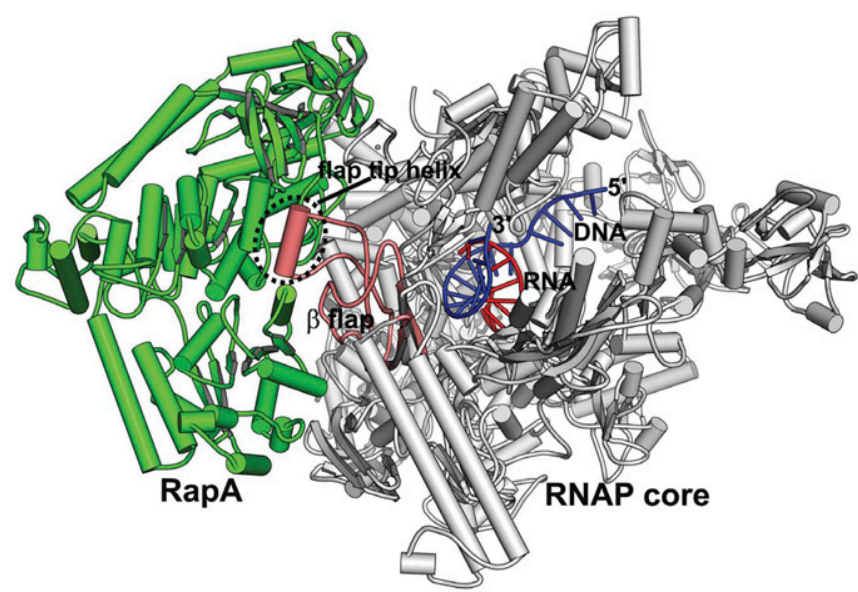

Fig. 1. Structure of RNAP core in complex with RapA and a DNA-RNA hybrid. An overview of the RNAP core-RapA-DNA-RNA complex. RapA is colored green; RNAP core is colored gray except for the $\beta$-flap domain, which is colored salmon; and the hybrid is colored blue (DNA) and red (RNA).

contacts with several structural domains that form the RNA exit channel (Fig. $2 A$ and $C$ ): the RapA NTD interacts with the $\beta$ zinc-binding domain (ZBD); the CTD of RapA interacts with both the C-terminal helix of the $\omega$-subunit and the $\mathrm{N}$ terminus of the $\beta^{\prime}$ subunit; the $2 \mathrm{~A} / 2 \mathrm{~B}$ lobe makes contacts with the $\beta$-flap domain and the 163-168 loop of the $\alpha_{\text {I }}$ subunit; and most significantly, the spacer domain of RapA is inserted into the RNA exit channel and makes contacts with the dock and ZBD domains of the $\beta^{\prime}$ subunit and the flap domain of the $\beta$-subunit (Fig. S3).

Unlike the RNAP holoenzyme, in which the RNA exit channel is completely blocked by the $\sigma_{3.2}$ linker of the $\sigma$-factor (19-21), the RapA-RNAP core complex exhibits an RNA channel that is narrowed by the insertion of the RapA spacer domain that partially blocks the RNA channel at the exit (Fig. 2D). The association of RapA with RNAP extends its RNA channel, which appears to exit at the groove space between the ZBD and the $\mathrm{N}$ terminus of the $\beta^{\prime}$ subunit. The remodeled RNA exit channel cannot accommodate an RNA hairpin inside the channel (Fig. S3). Consistent with this structural remodeling of the RNA exit channel, it was shown in an in vitro assay that the transcription termination efficiency at a hairpin-dependent terminator decreases in the presence of the RapA protein (22), suggesting that RapA plays a role in regulating transcription termination at intrinsic terminators that form RNA hairpins.

dsDNA Binding and DNA Translocation of Swi2/Snf2 Translocases. RapA binds both single-stranded (ss) and double-stranded (ds) polynucleotides and has a higher affinity for dsDNA (12). Both the sequence and structure of the ATPase core of RapA are highly similar to those of the homologous Sulfolobus solfataricus Rad54 (ssRad54) DNA translocase (13, 17, 23). A dsDNAbinding site could be identified on RapA by superposing the structure of the 1A domain of RapA onto the homologous domain of ssRad54 bound to dsDNA (23) (Fig. S4A). A similar superposition was also made with the UvrD helicase in complex with DNA (24) (Fig. S4B). As shown by aligning the corresponding 1A domains of ssRad54 and UvrD, single-stranded DNA binds to the UvrD helicase at the same site and in the same orientation as one of the dsDNA strands in the ssRad54 complex (Fig. S4C). Based on the similarity of DNA recognition and RecA-like architectures between Swi2/Snf2 enzymes and DExx box helicases, an ATP-driven translocation mechanism was derived for the Swi2/Snf2 proteins (23). According to this mechanism, dsDNA binds at the DNA-binding site of domain $1 \mathrm{~A}$ before translocation. The binding of ATP rearranges domain $2 \mathrm{~A}$ to push on the minor groove to advance dsDNA. Following DNA translocation, ATP hydrolysis relaxes the structure to allow rebinding of the enzyme at a new translocated DNA site. The displacement of the FTH, which interacts with both lobes of the ATPase core, may affect the dynamics of RapA conformational changes, and thus account for the change in ATPase activity of RapA upon its binding to RNAP.

Orientation of RapA Suggests Backtranslocation During Transcription. In a RapA-associated transcription elongation complex (TEC) (Fig. 3), the predicted dsDNA-binding site of RapA faces the upstream DNA of the complex. Taking into account the flexibility of the upstream DNA at the junction of the transcription bubble, it appears plausible that the upstream DNA of the TEC might swing to the dsDNA-binding site of RapA and form an elongation complex similar to the transcription initiation complex with upstream DNA bound to the C-terminal domain of the $\sigma$-factor (25). In this elongation complex, RapA interacts with the upstream DNA on one side, and with the upstream RNA exiting the RNA channel on the other side. Based on the mechanism described above for the Swi2/Snf2 translocases, the binding of ATP and its hydrolysis may move the RapA protein away from the transcription bubble when it binds the upstream DNA; in other words, due to the association of RapA with RNAP, the upstream DNA would be pulled back into the transcription bubble. This ATP-dependent backward translocation would resemble the RNAP translocation facilitated by the UvrD helicase that allows DNA repair enzymes to gain access to sites of damage in transcription-coupled DNA repair (11), whereas it is directly opposite to the translocation mediated by the mutation frequency decline ( $m f d$ ) gene product, another $E$. coli Swi2/ Snf2 dsDNA translocase that induces forward translocation of RNAP to rescue arrested and backtracked transcription elongation complexes or promote transcription termination $(26,27)$.

Mechanism and Function of RapA in Transcription Regulation. The idea that RapA actively backtracks transcribing RNAP appears counterintuitive, because active backtracking is opposite to the forward translocation of normal transcription. Excessive backtracking is believed to be harmful to genome stability and appears to be suppressed via various mechanisms in cellular organisms (10). However, compared with the typical transcription rate

Table 1. Data collection and refinement statistics

Parameters

RNAP-RapA complex

\begin{tabular}{lc}
\hline Data collection & $C 2$ \\
Space group & \\
Cell dimensions & $336.09,158.93,255.01$ \\
$a, b, c$ in $\AA$ & $90.00,101.28,90.00$ \\
$\alpha, \beta, \gamma\left(^{\circ}\right)$ & $49.53-4.70(4.95-4.70)$ \\
Resolution, $\AA *$ & $5.9(31.1)$ \\
$R_{\text {sym }}(\%)^{*}{ }^{*}$ & $6.7(2.0)$ \\
$l / \sigma^{*}$ & $99.4(99.1)$ \\
Completeness $(\%)^{*}$ & $2.4(2.4)$ \\
Redundancy* & \\
Refinement & $20-4.7$ \\
Resolution, $\AA$ & 60,931 \\
No. reflections & $27.34 / 36.93$ \\
$R_{\text {factor }} / R_{\text {free }}(\%)$ & 60,785 \\
No. atoms & 139.55 \\
$B$-factors & 0.007 \\
Rmsd bond, $\AA$ & 1.101 \\
Rmsd angle $\left({ }^{\circ}\right)$ &
\end{tabular}

*The highest resolution shell is shown in parentheses.

${ }^{\dagger} R_{\text {sym }}=\Sigma|\mathrm{I}-\langle\mathrm{l}\rangle| / \Sigma \mathrm{l}$, where $\mathrm{I}$ is the observed intensity and $\langle\mathrm{l}\rangle$ is the averaged intensity of multiple observation of symmetry-related reflections. 

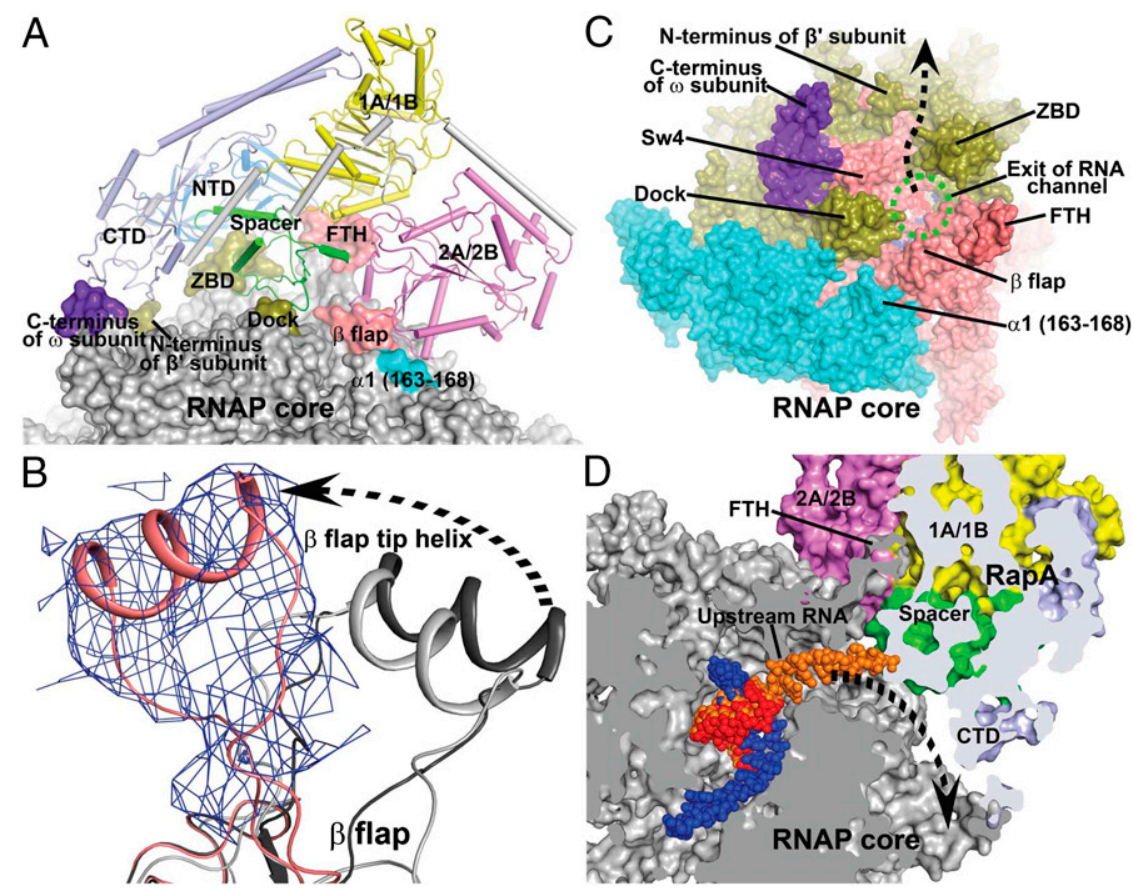

Fig. 2. Interactions between RNAP core and RapA. (A) Overview of the interface between RNAP core (surface representation) and RapA (tube and arrows). The contact areas on the RNAP core are labeled and highlighted with colors. The RapA domains NTD, 1A/1B, 2A/2B, spacer, and CTD are shown in marine, yellow, violet, green, and pale blue, respectively. $(B)$ Displacement of the $\beta$-flap tip helix in the RapA-RNAP core complex (salmon) relative to those in an RNAP holoenzyme (dark gray, PDB code 4JKR) (21) and an elongation complex (light gray, PDB code 205I) (16). Unbiased $2 F_{\mathrm{o}}-F_{\mathrm{c}}$ density map (contoured at $1 \sigma$ ) is shown as blue meshes for the FTH in the RapA-RNAP core complex. (C) Viewing down the RNA exit channel (green dashed oval) of RNAP. RapA is omitted for clarity. The RNAP core is shown in surface representation. Regions around the RNA exit channel are labeled. The dashed arrow marks the putative new RNA exit direction. $(D)$ Clip view of the RNA exit channel. Both RNAP core and RapA are shown in surface representations. The RNAP core is colored in gray and RapA domains are colored as in A. RNA residues from a transcription elongation complex (16) are superimposed on the DNA-RNA hybrid and shown in orange for reference. A dashed arrow marks the potential RNA exit in the complex. FTH, $\beta$-flap tip helix; ZBD, $\beta^{\prime}$ zinc-binding domain; SW4, RNAP switch 4; dock, $\beta^{\prime}$ dock domain.

of $20-80 \mathrm{nt} / \mathrm{s}$, the ATP hydrolysis by RapA $\left(\mathrm{k}_{\mathrm{cat}}<1 \mathrm{~s}^{-1}\right)(12)$ is a much slower process. The relatively weak DNA affinity of $\operatorname{RapA}\left(\mathrm{K}_{\mathrm{d}} \sim 20 \mu \mathrm{M}\right)(12)$ and the slow ATP hydrolysis implies that backtracking mediated by RapA is stochastic. During normal transcription elongation, the weak-DNA binding RapA might rarely have a chance to exert a force on the DNA, because the fast-moving

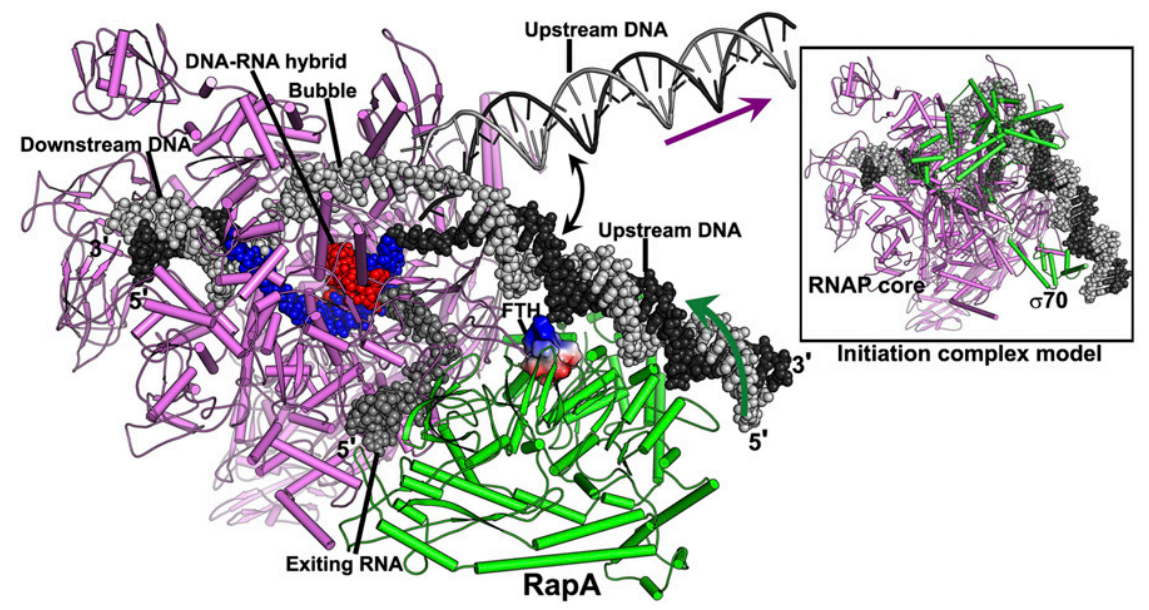

Fig. 3. A model of the transcription elongation complex in the presence of RapA. The portions of nucleic acids colored in red (RNA) and in blue (DNA) are from this study. The downstream DNA (black and gray space-filling model) was modeled using a Thermus thermophilus elongation complex (16). The movable upstream DNA (black and gray ladders) is a hypothetical model for an elongation complex by assuming that a 12-bp transcription bubble connects the upstream dsDNA (modeled with idea B-form DNA) and the downstream dsDNA. The RapA-interacting upstream DNA (space-filling representation) was modeled by extending a piece of dsDNA (placed by superimposing homologous structures as shown in Fig. S4) to connect with the transcription bubble. A hypothetical model of the exiting RNA is displayed in space-filling form (dark gray) to show the altered RNA exit channel. The flap tip helix (FTH) of the RNAP is shown as an electrostatic surface potential diagram. The dark green arrow marks the direction of DNA translocation promoted by RapA, which is opposite to the normal direction of DNA translocation during transcription (purple arrow). The Inset is a model of the transcription initiation complex (25) shown in the same orientation for comparison. The core RNAP is colored in violet, and both RapA and the $\sigma^{70}$ factor are colored in green. 
RNAP would readily dissociate any accidentally associated RapA from the upstream DNA before RapA gets to hydrolyze an ATP. Active backtracking would become significant only when transcription is paused, a process that happens every few hundred nucleotides during transcription and is enhanced upon nucleotide misincorporation or by other signals (3). Although this ATPdriven backtranslocation is predicted to be a slow stochastic process, it would still be much more efficient than random walking when extensive backtracking is necessary.

The ATP-driven backward translocation of RNAP can explain the in vitro ability of RapA bound to stalled RNAP to stimulate RNA synthesis $(13,15)$ (Fig. 4). In the case of the accumulation of backtracked RNAP complexes due to insufficient RNA cleavage, continuous backtracking would release RNA through the secondary channel to recycle the enzyme, whereas for transcription stalled at hypertranslocated states, RNA synthesis might be able to resume as the RNA $3^{\prime}$ end is brought back to the RNAP catalytic center by the RapA-mediated backward movement. Even when strong secondary structures exist on the upstream RNA, the ATP-powered machinery may still be able to function like a helicase to unfold RNA structures as it translocates backward.
However, it might be worth noting that helicase activity has not been detected to date and is also not expected for the RapA protein alone. It is possible that RapA functions to reactivate RNA polymerases in vivo as well, although its role has not been established possibly due to existence of functionally redundant processes.

Backtranslocation as a General Mechanism of Transcription Regulation. Cellular RNAPs translocate back and forth via a thermal ratchet mechanism $(1,28)$, and external energy input is necessary to shift the thermodynamic equilibrium to favor translocation in either direction. When transcription is arrested in a sequence-specific manner or by other causes such as DNA lesions, the deadlock can be resolved by ATP-driven hypertranslocation or backtracking. Hypertranslocation, as exemplified by mfd (26), terminates transcription, whereas backtracking, such as that shown recently for UvrD (11), temporarily moves the RNAP away from the $3^{\prime}$ end of the growing RNA and would allow transcription to resume later. The ATP-driven process proposed here for RapA action also promotes translocation of RNAP in the reverse direction, which might be triggered by the ubiquitous transcriptional pausing.

A
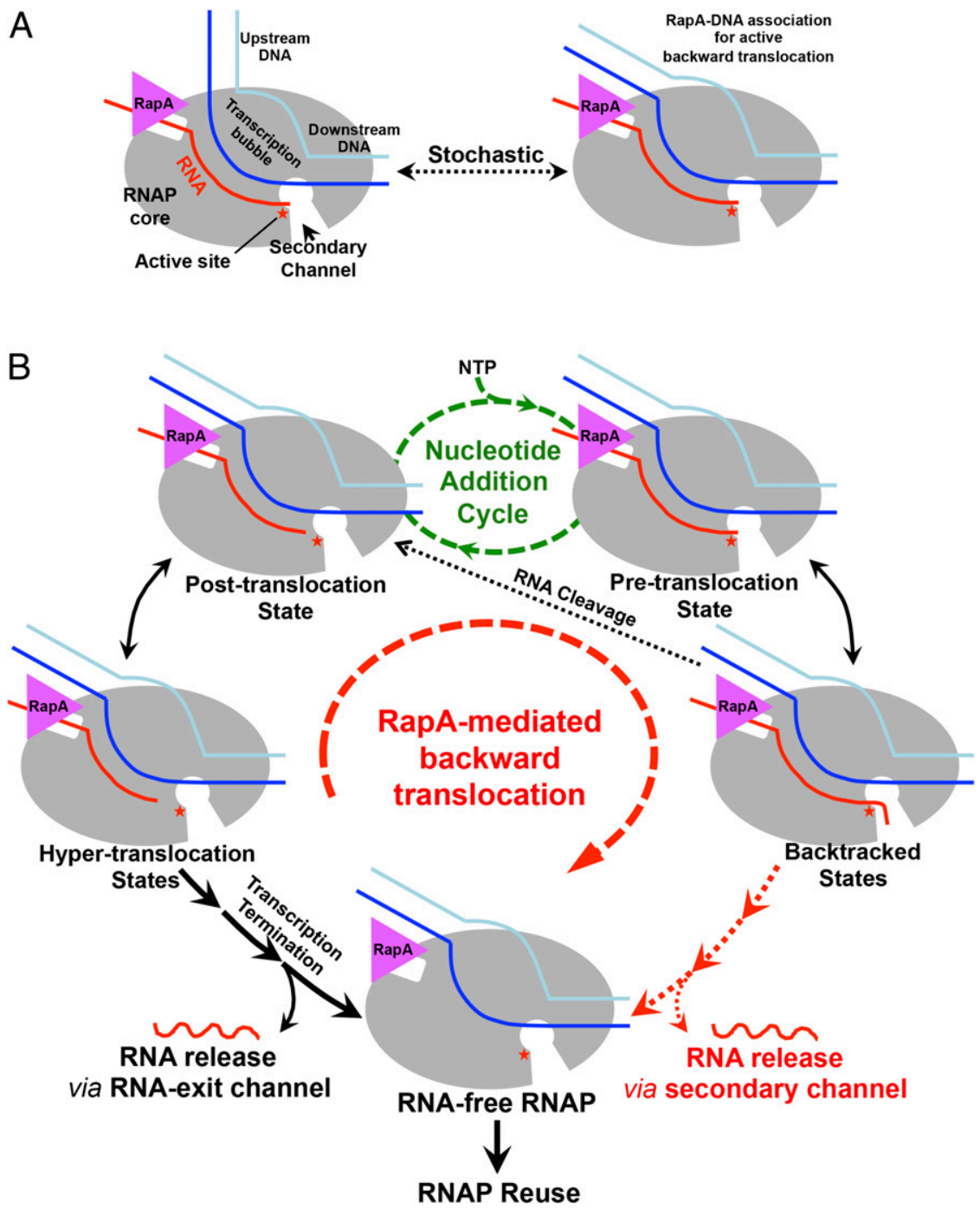

Fig. 4. Schematics of the proposed mechanism of RNAP reactivation by RapA. $(A)$ Stochastic switching of the upstream DNA between the free and RapAinteracting forms. $(B)$ When the upstream DNA interacts with RapA, the ATPase activity of RapA would power backward translocation of RNAP. Backtranslocation of hypertranslocated RNAP would bring the RNA $3^{\prime}$ end back to the RNAP active center for transcription to resume, whereas backtracked RNAP might be reactivated by RNA cleavage or be recycled with continued backtranslocation to release RNA via the secondary channel. 
This process would not only be compatible with the RNA cleavage of backtracked residues, but would also be able to reverse hypertranslocation to reactivate transcription. It is conceivable that RapA could also function like the UvrD helicase to backtrack stalled RNAP in transcription-coupled DNA repair. It has been shown that an $E$. coli strain with a disruption of the rap $A$ gene displays sensitivity to UV light (14), although contradictory results have also been reported (29). Considering the plethora of Swi2/Snf2 family transcription regulators in all cellular organisms (18), active backward translocation, complementing the induced forward translocation, is likely a general mechanism for regulating transcription and transcription-related processes in both prokaryotes and eukaryotes.

\section{Materials and Methods}

Preparation of the RapA-RNAP Core Complex. The $E$. coli RNAP core enzyme and the $\mathrm{N}$-terminal his-tagged RapA protein were overexpressed and purified separately as described $(13,21)$. The purified RNAP core enzyme was mixed with an excess of RapA and loaded onto a Superdex G200 prep grade gel filtration column (GE Healthcare) equilibrated in the buffer ( $20 \mathrm{mM}$ Tris $\mathrm{pH} 7.5,50 \mathrm{mM} \mathrm{NaCl}, 0.1 \mathrm{mM}$ EDTA, $5 \mathrm{mM} \mathrm{MgCl}$ ). The fractions containing the RapA-RNAP core complex were pooled and concentrated to around $20 \mathrm{mg} \cdot \mathrm{ml}^{-1}$.

Crystallization of the RapA-RNAP Core Complex. The core RNAP-RapA-DNARNA complex was formed by directly mixing the RapA-RNAP core complex with a threefold molar excess of the preformed DNA-RNA hybrid (DNA sequence: 5'-ACGACTGAGCCGATG-3'; RNA sequence: 5'-AUCGGCUCA-3') at

1. Guajardo R, Sousa R (1997) A model for the mechanism of polymerase translocation. J Mol Biol 265(1):8-19.

2. Martinez-Rucobo FW, Cramer P (2013) Structural basis of transcription elongation. Biochim Biophys Acta 1829(1):9-19.

3. Landick R (2006) The regulatory roles and mechanism of transcriptional pausing Biochem Soc Trans 34(Pt 6):1062-1066.

4. Neuman KC, Abbondanzieri EA, Landick R, Gelles J, Block SM (2003) Ubiquitous transcriptional pausing is independent of RNA polymerase backtracking. Cell 115(4): 437-447.

5. Adelman K, et al. (2002) Single molecule analysis of RNA polymerase elongation reveals uniform kinetic behavior. Proc Natl Acad Sci USA 99(21):13538-13543.

6. Artsimovitch I, Landick R (2000) Pausing by bacterial RNA polymerase is mediated by mechanistically distinct classes of signals. Proc Natl Acad Sci USA 97(13):7090-7095.

7. Roberts J, Park JS (2004) Mfd, the bacterial transcription repair coupling factor: Translocation, repair and termination. Curr Opin Microbiol 7(2):120-125.

8. Peters JM, Vangeloff AD, Landick R (2011) Bacterial transcription terminators: The RNA 3'-end chronicles. J Mol Biol 412(5):793-813.

9. Sydow JF, Cramer P (2009) RNA polymerase fidelity and transcriptional proofreading. Curr Opin Struct Biol 19(6):732-739.

10. Nudler E (2012) RNA polymerase backtracking in gene regulation and genome instability. Cell 149(7):1438-1445.

11. Epshtein V, et al. (2014) UvrD facilitates DNA repair by pulling RNA polymerase backwards. Nature 505(7483):372-377.

12. Sukhodolets MV, Jin DJ (1998) RapA, a novel RNA polymerase-associated protein, is a bacterial homolog of SWI2/SNF2. J Biol Chem 273(12):7018-7023.

13. Shaw G, et al. (2008) Structure of RapA, a Swi2/Snf2 protein that recycles RNA polymerase during transcription. Structure 16(9):1417-1427.

14. Muzzin O, et al. (1998) Disruption of Escherichia coli hepA, an RNA polymeraseassociated protein, causes UV sensitivity. J Biol Chem 273(24):15157-15161.

15. Sukhodolets MV, Cabrera JE, Zhi H, Jin DJ (2001) RapA, a bacterial homolog of SWI2/ SNF2, stimulates RNA polymerase recycling in transcription. Genes Dev 15(24): 3330-3341.

16. Vassylyev DG, Vassylyeva MN, Perederina A, Tahirov TH, Artsimovitch I (2007) Structural basis for transcription elongation by bacterial RNA polymerase. $\mathrm{Na}$ ture 448(7150):157-162.

17. Bork P, Koonin EV (1993) An expanding family of helicases within the 'DEAD/H' superfamily. Nucleic Acids Res 21(3):751-752.

18. Flaus A, Martin DM, Barton GJ, Owen-Hughes T (2006) Identification of multiple distinct Snf2 subfamilies with conserved structural motifs. Nucleic Acids Res 34(10): 2887-2905. room temperature. This mixture was then used directly for crystallization at room temperature by vapor diffusion with $5.2 \%$ (wt/vol) PEG 3350, $50 \mathrm{mM}$ $\mathrm{MgCl}_{2}$, and $100 \mathrm{mM}$ Hepes $\mathrm{pH}$ 7.0. After crystals grew to full size for about a week, they were cryoprotected in mother liquor containing 30\% (wt/vol) ethylene glycol before flash frozen in liquid nitrogen.

Data Processing and Structure Determination. X-ray diffraction data were collected at $100 \mathrm{~K}$ at the beamline $\mathrm{X} 25$ at Brookhaven National Laboratory. All data were integrated and scaled using IMOSFLM (30) and SCALA (31). The crystal belongs to the centered monoclinic space group C2 with two copies of the complex per asymmetric unit. The structure of the core RNAP-RapADNA-RNA complex was solved by molecular replacement with PHASER (32) using structures of the core portions of the $E$. coli RNAP holoenzyme (21) and the RapA (13) protein as the searching models. Phases were improved by noncrystallographic symmetry (NCS) averaging between the two copies within the asymmetric unit using the program DM (33) in the CCP4 suite (34). After building the DNA-RNA hybrid based on the unbiased average map in COOT (35), translation, liberation, and screw rotation (TLS) and NCS restrained refinement were performed using REFMAC5 (36) to generate the final structural model. The structure was refined to a resolution of $4.7 \AA(I / \sigma=2)$ with an $R_{\text {work }} / R_{\text {free }}$ of $0.273 / 0.369$. Data collection and structural refinement statistics are summarized in Table 1. All figures were created using PyMOL (37).

ACKNOWLEDGMENTS. We thank D. J. Jin (NIH) for the E. coli RapA-overexpressing plasmid, the staff of the National Synchrotron Light Source Beamline X25 for help during data collection, and the Center for Structura Biology facility at Yale University for computational support. This work was supported by NIH Grant GM57510 (to T.A.S.). T.A.S. is an investigator of the Howard Hughes Medical Institute.

19. Murakami KS, Masuda S, Darst SA (2002) Structural basis of transcription initiation: RNA polymerase holoenzyme at 4 A resolution. Science 296(5571):1280-1284.

20. Vassylyev DG, et al. (2002) Crystal structure of a bacterial RNA polymerase holoenzyme at 2.6 A resolution. Nature 417(6890):712-719.

21. Zuo Y, Wang Y, Steitz TA (2013) The mechanism of E. coli RNA polymerase regulation by ppGpp is suggested by the structure of their complex. Mol Cell 50(3):430-436.

22. Yawn B, Zhang L, Mura C, Sukhodolets MV (2009) RapA, the SWI/SNF subunit of Escherichia coli RNA polymerase, promotes the release of nascent RNA from transcription complexes. Biochemistry 48(33):7794-7806.

23. Dürr H, Körner C, Müller M, Hickmann V, Hopfner KP (2005) X-ray structures of the Sulfolobus solfataricus SWI2/SNF2 ATPase core and its complex with DNA. Cell 121(3):363-373.

24. Lee JY, Yang W (2006) UvrD helicase unwinds DNA one base pair at a time by a twopart power stroke. Cell 127(7):1349-1360.

25. Hudson BP, et al. (2009) Three-dimensional EM structure of an intact activatordependent transcription initiation complex. Proc Natl Acad Sci USA 106(47): 19830-19835.

26. Park JS, Marr MT, Roberts JW (2002) E. coli Transcription repair coupling factor (Mfd protein) rescues arrested complexes by promoting forward translocation. Cell 109(6): 757-767.

27. Deaconescu AM, et al. (2006) Structural basis for bacterial transcription-coupled DNA repair. Cell 124(3):507-520.

28. Bar-Nahum G, et al. (2005) A ratchet mechanism of transcription elongation and its control. Cell 120(2):183-193.

29. Sukhodolets MV, Jin DJ (2000) Interaction between RNA polymerase and RapA a bacterial homolog of the SWI/SNF protein family. J Biol Chem 275(29):22090-22097.

30. Leslie AG (1999) Integration of macromolecular diffraction data. Acta Crystallogr D Biol Crystallogr 55(Pt 10):1696-1702.

31. Evans P (2006) Scaling and assessment of data quality. Acta Crystallogr D Biol Crystallogr 62(Pt 1):72-82.

32. McCoy AJ, et al. (2007) Phaser crystallographic software. J App/ Cryst 40(Pt 4):658-674

33. Cowtan K (1999) Error estimation and bias correction in phase-improvement calculations. Acta Crystallogr D Biol Crystallogr 55(Pt 9):1555-1567.

34. Winn MD, et al. (2011) Overview of the CCP4 suite and current developments. Acta Crystallogr D Biol Crystallogr 67(Pt 4):235-242.

35. Emsley P, Cowtan K (2004) Coot: Model-building tools for molecular graphics. Acta Crystallogr D Biol Crystallogr 60(Pt 12 Pt 1):2126-2132.

36. Murshudov GN, et al. (2011) REFMAC5 for the refinement of macromolecular crystal structures. Acta Crystallogr D Biol Crystallogr 67(Pt 4):355-367.

37. DeLano WL (2002) The PyMOL Molecular Graphics System (DeLano Scientific, San Carlos, CA). 